\title{
ON STRONG COUPLING THEORY OF CORRELATED SUPERCONDUCTORS
}

\author{
K.I. WỴsoKIŃSKI \\ Institute of Physics, Maria Curie-Skłodowska University \\ Radziszewskiego 10A, 20-031 Lublin, Poland
}

\begin{abstract}
The strong coupling or Eliashberg equations of superconductivity have been generalised to describe strongly correlated systems. Calculations show that $d$-wave superconductivity is stable for small hole doping. It also follows that in correlated systems one should define three different Eliashberg functions and corresponding electron-phonon coupling constants $\lambda_{\mathrm{N}}, \lambda_{\mathrm{S}}$ and $\lambda_{\mathrm{tr}}$ (to describe normal (N), superconducting (S) and transport (tr) properties) each of which is measured in different kind of experiment.
\end{abstract}

PACS numbers: 74.20.-z, 74.20.Mn, 74.25.Kc

Recently an increased interest is observed in the study of mutual interplay between electron-phonon and short range electron-electron interaction. As a model system the superconducting oxides may serve. They are strongly correlated systems in which the electron-phonon interaction does play an important role [1-3].

In this work we are mainly interested in some general properties of phonon induced superconductivity in correlated systems. It is thus instructive to start with a model which is simple and at the same time captures the main physics of such materials. The proper model is the usual Hubbard model supplemented with terms describing the electron-phonon interaction. Both the covalent and ionic parts of the interaction are taken into account. For our purposes it is enough to treat strong correlations via slave boson method in the $U \rightarrow \infty$ limit [4].

Denoting fermion, auxiliary boson and phonon creation (annihilation) operators by $c_{i \sigma}^{+}, b_{i}^{+}, a_{q \nu}^{+}\left(c_{i \sigma}, b_{i}, a_{q \nu}\right)$, respectively, we write the Hamiltonian of the system in the form

$$
\begin{gathered}
H=\sum_{i j \sigma} t_{i j} c_{i \sigma}^{+} c_{j \sigma} b_{i} b_{j}^{+}+\sum_{i} \Lambda_{i}\left(\sum_{\sigma} c_{i \sigma}^{+} c_{i \sigma}+b_{i}^{+} b_{i}-1\right)+\sum_{i j s \sigma \alpha} T_{i j s}^{\prime \alpha} u_{s}^{\alpha} c_{i j}^{+} c_{j \sigma} b_{i} b_{j}^{+} \\
+\sum_{i s \alpha} V_{i s}^{\alpha} u_{s}^{\alpha} c_{i \sigma}^{+} c_{i \sigma}-\mu \sum_{i \sigma} c_{i \sigma}^{+} c_{i \sigma}+\sum_{q \nu} \hbar \omega_{q \nu}\left(a_{q \nu}^{+} a_{q \nu}+\frac{1}{2}\right)
\end{gathered}
$$

In order to properly take into account the absence of doubly occupied sites it is important to work in site representation. It turns out that no-double occupancy requirement does not modify the equations for the normal parts of the self-energy, 
which contain the factors of the type $\left\langle c_{i \sigma}^{+} c_{j \sigma}\right\rangle$. They are allowed even for $i=j$. Contrary to that the anomalous part of the self-energy depends on correlation function $\left\langle c_{i \sigma}^{+} c_{j-\sigma}^{+}\right\rangle$, which for $i=j$ describes the amplitude of a pair at the $i$-th site, and should vanish in strongly correlated systems. This important fact has been first noted by Zielinski and coworkers [2] and leads to severe changes in the form of Eliashberg equations [5].

In the mean field approximation for slave bosons one obtains the real frequency axis Eliashberg equations in the following form [5]:

$$
\begin{aligned}
& \omega\left[1-Z_{k}(\omega)\right]= \\
& \frac{1}{2} \int \mathrm{d} \omega_{1} \int \mathrm{d} \omega_{2} \frac{\operatorname{th} \frac{\beta \omega_{1}}{2}+\operatorname{cth} \frac{\beta \omega_{2}}{2}}{\omega-\omega_{1}-\omega_{2}} \frac{1}{\mathrm{~N}} \sum_{k^{\prime}} K_{k k^{\prime}}^{\mathrm{N}}\left(\omega_{2}\right)\left(-\frac{1}{\pi}\right) \operatorname{Im} \frac{\omega_{1} Z_{k^{\prime}}\left(\omega_{1}\right)}{D_{k^{\prime}}\left(\omega_{1}\right)}, \\
& \phi_{k}(\omega)= \\
& \frac{1}{2} \int \mathrm{d} \omega_{1} \int \mathrm{d} \omega_{2} \frac{\operatorname{th} \frac{\beta \omega_{1}}{2}+\operatorname{cth} \frac{\beta \omega_{2}}{2}}{\omega-\omega_{1}-\omega_{2}} \frac{1}{\mathrm{~N}} \sum_{k^{\prime}} K_{k k^{\prime}}^{\mathrm{S}}\left(\omega_{2}\right)\left(-\frac{1}{\pi}\right) \operatorname{Im} \frac{-\phi_{k^{\prime}}\left(\omega_{1}\right)}{D_{k^{\prime}}\left(\omega_{1}\right)},
\end{aligned}
$$

where we have denoted $D_{k^{\prime}}\left(\omega_{1}\right)=\left[\omega_{1} Z_{k^{\prime}}\left(\omega_{1}\right)\right]^{2}-\left[\phi_{k^{\prime}}\left(\omega_{1}\right)\right]^{2}-\left[r^{2} \epsilon_{k^{\prime}}-\mu+\Lambda+\right.$ $\left.\chi_{\boldsymbol{k}^{\prime}}\left(\omega_{1}\right)\right]^{2}$ and

$$
\begin{aligned}
K_{k k^{\prime}}^{\mathrm{N}}\left(\omega_{2}\right) & =\sum_{\nu}\left|M_{k, k-k^{\prime}}^{\nu}\right|^{2}\left(-\frac{1}{\pi}\right) \operatorname{Im}\left\langle\left\langle\phi_{k-k^{\prime}, \nu} \mid \phi_{-k+k^{\prime}, \nu}\right\rangle\right\rangle_{\omega_{2}+i 0} \\
K^{\mathrm{S}}{ }_{k, k^{\prime}}\left(\omega_{2}\right) & =\frac{1}{N} \sum_{q_{\nu}}\left|M_{k, q}^{\nu}\right|^{2}\left(-\frac{1}{\pi}\right) \operatorname{Im}\left\langle\left\langle\phi_{q, \nu} \mid \phi_{-} q_{\nu}\right\rangle\right\rangle_{\omega_{2}+\mathrm{i} 0} \gamma\left(k-q-k^{\prime}\right) .
\end{aligned}
$$

$M_{k, q}^{\nu}$ is the electron-phonon matrix element [5], $r^{2}=\left\langle b_{i}^{+}\right\rangle\left\langle b_{i}\right\rangle=1-n$ and $n$ is the electron concentration.

Two different kernels $K_{k, k^{\prime}}^{\mathrm{N}}$ and $K_{k, k^{\prime}}^{\mathrm{S}}$ of Eliashberg equations imply the existence of two different Eliashberg functions $\alpha^{2} F_{\mathrm{I}}(\omega), \quad I=N, \mathrm{~S}$ for normal (N) and superconducting (S) properties defined by

$$
\alpha^{2} F_{\mathrm{I}}(\omega)=N\left(E_{\mathrm{F}}\right)\left\langle\left\langle K_{k, k^{\prime}}^{\mathrm{I}}(\omega)\right\rangle_{k_{\mathrm{F}}^{\prime}}\right\rangle_{k_{\mathrm{F}}}, \quad \mathrm{I}=\mathrm{N}, \mathrm{S},
$$

where the symbol $\left\langle\langle\cdots\rangle_{k_{\mathrm{F}}}\right\rangle_{\boldsymbol{k}_{\mathrm{F}}^{\prime}}$ denotes an average over the Fermi surface average and $N\left(E_{\mathrm{F}}\right)$ is the density of states at the Fermi energy. One also defines two different electron-phonon coupling constants $\lambda_{\mathrm{I}}=2 \int \frac{\mathrm{d} \omega}{\omega} \alpha^{2} F_{\mathrm{I}}(\omega)$.

For completeness let us remind that there exists [6] still another Eliashberg function which in the present case can be defined as

$$
\alpha^{2} F_{\mathrm{tr}}(\omega)=N\left(E_{\mathrm{F}}\right)\left\langle\left\langle K_{k, k^{\prime}}^{\mathrm{N}}(\omega) \frac{\left[v(k)-v\left(k^{\prime}\right)\right]^{2}}{2\left\langle v(k)^{2}\right\rangle_{k_{\mathrm{F}}}}\right\rangle_{k_{\mathrm{F}}^{\prime}}\right\rangle_{k_{\mathrm{F}}},
$$

and corresponding coupling $\lambda_{t r}$ which describes transport (tr) properties of the system.

In Fig. 1 we show the concentration dependence of the total superconducting coupling constant $\lambda_{\mathrm{S}}$ (solid line) and its various symmetry components. We have studied $s, p$ and $d$-wave symmetries. We have used the full wave vector dependence 


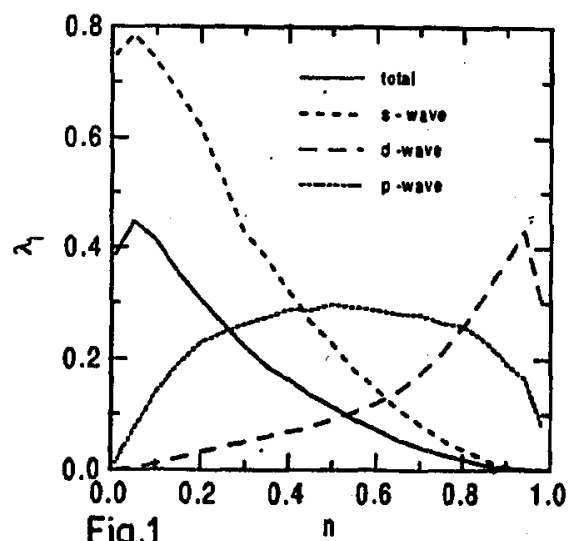

Fig.1

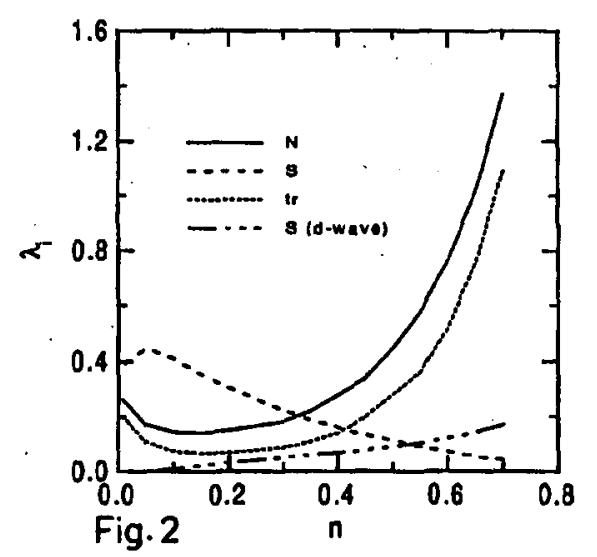

Fig. 1. The concentration dependence of the superconducting coupling constant (solid line) and its $s, p$ and $d$-wave symmetry components.

Fig. 2. The concentration dependence of the various coupling constants: $\lambda_{\mathrm{N}}$ (solid line), $\lambda_{\mathrm{S}}$ (dashed line), $\lambda_{\mathrm{tr}}$ (dotted line). Dashed-dotted line shows $d$-wave component of $\lambda_{\mathrm{S}}$.

of the electron-(optical)phonon matrix elements and values of other parameters appropriate for oxide superconductors. Both the covalent and ionic type of the electron-phonon interaction contribute to the coupling. Note that in correlated systems the $d$-wave component of the coupling dominates at low hole doping. This indicates a possibility of the $d$-wave superconductivity from the electron-phonon interaction.

In Fig. 2 we show the dependence on carrier concentration of the various coupling constants $\lambda_{\mathrm{I}}$, calculated for a mixed (covalent plus ionic) type of the electron-phonon interaction. Solid, dashed and dotted lines in the figure refer to a normal, superconducting and transport coupling constant, respectively. A dashed-dotted line represents $d$-wave component of the superconducting coupling.

The important message from these calculations is that in correlated systems the relative values of coupling constants depend on carrier concentration and also on the details of wave vector dependence of the electron-phonon matrix elements $M_{k \cdot k^{\prime}}$

In all cases we have studied and for carrier concentration in the range of interest for high $T_{\mathrm{c}}$ superconductors the transport coupling is always larger than the normal or superconducting one. This is at odds with usual claims that the measured $\lambda_{t r}$ is smaller than $\bar{\lambda}$ deduced from the superconducting transition temperature. Certainly the vertex corrections (see Kulić and Zeyher in Ref. [1]) are important for a quantitative comparison between the experiment and theory.

In conclusion we have found that three different electron-phonon coupling parameters are necessary to described correlated systems. In particular both $\lambda_{\mathrm{S}}$ and $\lambda_{\mathrm{N}}$ enter the formula for superconducting transition temperature. 


\section{Acknowledgments}

A part of this work has been done during author's stay at ISI Torino supported by the EC contract ERBIPDCT 940027.

\section{References}

[1] Ju H. Kim, Z. Tešanović, Phys. Rev. Lett. 71, 4218 (1993); ibid. 74, 1489 (1995); M. Grilli, C. Castellani, Phys. Rev. B 50, 16880 (1994); Phys. Rev. Lett. 74, 1448 (1995); M. Mierzejewski, J. Zieliński, Phys. Rev. B 52, 3079 (1995); M.L. Kulić, R. Zeyher, Phys. Rev. B 49, 4395 (1994).

[2] J. Zieliński, M. Mierzejewski, P. Entel, R. Grabowski, J. Supercond. 8, 135 (1995).

[3] K.A. Muller, Z. Phys. B 80, 193 (1990).

[4] D.M. Newns, N. Read, Adv. Phys. 36, 799 (1987).

[5] K.I. Wysokiński, Phys. Rev. B 54, 3553 (1996).

[6] G. Grimvall, The Electron-Phonon Interaction in Metals, North-Holland, Amsterdam 1981, p. 4. 